\title{
CONTROLE FUZZY MULTIOBJETIVO DE SISTEMAS DINÂMICOS NÃO-LINEARES COM ATRASO PURO DE TEMPO
}

\author{
Rafael J. M. Santos*, Ginalber L. O. Serra* \\ *Instituto Federal de Educação, Ciência e Tecnologia do Maranhão \\ Departamento de Eletroeletrônica \\ Av. Getúlio Vargas, N. 04, Monte Castelo, São Luís, Maranhão, Brazil \\ Emails: rafael82ms@yahoo.com.br, ginalber@ifma.edu.br
}

\begin{abstract}
In this paper a method of optimal pole placement state-space Takagi-Sugeno (T-S) fuzzy control for nonlinear systems with time delay is proposed. The techniques of pole placement and linear quadratic regulator $(\mathrm{LQR})$ are combined in a fuzzy context in order to ensure an optimal controller with satisfactory performance based on temporal poles properly allocated and whose design takes into account the pure time delay of the plant be controlled. Experimental results for temperature LQR fuzzy, via data acquisition platform, in real time, according to the proposed methodology, show the efficiency and flexibility of design.
\end{abstract}

Keywords - Fuzzy control, LQR, pole placement, optimal control.

Resumo- Neste artigo é proposto um método de controle fuzzy Takagi-Sugeno (T-S) ótimo com alocação de pólos no espaço de estados para sistemas não-lineares com atraso puro de tempo. As técnicas de alocação de pólos e do regulador linear quadrático (LQR) são combinadas em um contexto fuzzy de maneira a garantir um controlador ótimo com desempenho temporal satisfatório baseado em pólos adequadamente alocados e cujo projeto leva em conta o atraso puro de tempo da planta a ser controlada. Resultados experimentais para controle LQR fuzzy de temperatura, via plataforma de aquisição de dados, em tempo real, de acordo com a metodologia proposta, mostram a eficiência e a flexibilidade de projeto.

Keywords - Controle fuzzy, LQR, alocação de pólos, controle ótimo.

\section{Introdução}

A teoria de sistemas fuzzy tem recebido especial atenção por parte dos pesquisadores na área de controle, por ser capaz de tratar eficientemente com incertezas e não-linearidades através da sua estrutura funcional. Este crescente interesse pelo desenvolvimento de métodos de projeto de controladores fuzzy se deve à notória possibilidade se incorporar na estrutura do controlador fuzzy o conhecimento do especialista bem como o formalismo matemático advindo da teoria de controle moderna. (Lendek et al., 2009; Michels et al., 2006; Babuška, 1998). Na literatura técnica existem diversas propostas e aplicações bem sucedidas de sistemas de controle fuzzy com estas características.Em (Jahromi and Zabihollah, 2010), é proposto um sistema de controle semi-ativo para sistemas de suspensão de automóveis que utiliza amortecedor magnetoreológico (MR), onde o mecanismo de controle foi desenvolvido com base no Regulador Linear Quadrático (LQR) e controlador fuzzy, o que, segundo os autores, possibilitou redução significativa na resposta do veículo de passageiros quanto a vibrações e também eliminação do efeito da perturbação do sistema, proporcionando uma condução confortável. Neste artigo é proposto um método de controle fuzzy Takagi-Sugeno (T-S) ótimo com alocação de pólos no espaço de estados para sistemas não-lineares com atraso puro de tempo. A alocação de pólos é uma técnica na qual todos os pólos de malha-fechada são alocados nas posições dese- jadas de modo a garantir que a resposta do sistema apresente aspectos previamente estabelecidos, como especificações da resposta temporal, coeficiente de amortecimento, máximo de sobresinal, como também especificações de regime permanente. Como técnica de controle ótimo é utilizado o regulador linear quadrático (LQR), que permite calcular de maneira sistemática a matriz de ganho de controle por realimentação de estado para o sistema de controle. Essas duas técnicas serão aqui combinadas em um contexto fuzzy de maneira a garantir um controlador ótimo com desempenho temporal satisfatório baseado em pólos adequadamente alocados e cujo projeto leva em conta o atraso puro de tempo da planta a ser controlada. Resultados experimentais para controle LQR fuzzy de temperatura, via plataforma de aquisição de dados, em tempo real, de acordo com a metodologia proposta, mostram a eficiência e a flexibilidade de projeto.

\section{Controle Fuzzy ótimo com alocação de pólos para sistemas com atraso de tempo}

O método do regulador linear quadrático $(L Q R)$ permite calcular sistematicamente a matriz de ganho de controle por realimentação de estado $\left(\mathbf{K}_{i}\right)$, referente ao $i$-ésimo submodelo do sistema fuzzy, a partir da função de custo estabelecida. A alocação de pólos, por sua vez, determina a matriz $\mathbf{K}_{i}$ que aloque os pólos desejados. Nesta seção será proposta uma associação entre essas duas aborda- 
gens, no contexto fuzzy, de modo que seja possível alocar os pólos de malha-fechada nas posições desejadas e ao mesmo tempo garantir um controle ótimo, considerando sistemas com atraso puro de tempo.

\subsection{Planta dinâmica nebulosa Takagi-Sugeno}

Um sistema de inferência fuzzy Takagi-Sugeno (TS) usa no consequente de suas regras SE-ENTÃO uma expressão funcional da variáveis linguísticas do antecedente. A $\left.i\right|^{[i=1,2, \ldots, l]}$-ésima regra TS, indicada por $R^{i}$, onde $l$ é o número de regras, é dada por

$$
\begin{aligned}
R^{i}: & \mathbf{S E} \tilde{x}_{1} e^{\prime} F_{j \mid \tilde{x}_{1}}^{i} \mathbf{E} \ldots \mathbf{E} \tilde{x}_{n} e^{\prime} F_{j \mid \tilde{x}_{n}}^{i} \\
& \mathbf{E N T} \tilde{\mathbf{A} O} \dot{\mathbf{x}}(t)=A_{i} \mathbf{x}(t)+B_{i} \mathbf{u}(t)
\end{aligned}
$$

$\mathrm{O}$ vetor $\tilde{\mathbf{x}} \in \mathbb{R}^{n}$ contem as variáveis linguísticas do antecedente. Cada variável linguística tem seu próprio universo de discurso $U_{\tilde{x}_{1}}, \ldots, U_{\tilde{x}_{n}}$ particionado pelos conjuntos fuzzy representando os termos linguísticos correspondentes. A variável $\left.\tilde{x}_{t}\right|^{t=1,2, \ldots,,}$, pertence ao conjunto fuzzy $F_{j \mid \tilde{x}_{t}}^{i}$ com um valor $\gamma_{F_{j \mid \tilde{x}_{t}}}^{i}$ definido por uma função de pertinência $\gamma_{\tilde{x}_{t}}^{i}: \mathbb{R} \rightarrow[0,1]$, com $\gamma_{F_{j \mid \tilde{x}_{1}}}^{i} \in$ $\left\{\gamma_{F_{1 \mid \tilde{x}_{1}}}^{i}, \gamma_{F_{2 \mid \tilde{x}_{1}}}^{i}, \ldots, \gamma_{F_{p_{\tilde{x}_{t}} \mid \tilde{x}_{1}}}^{i}\right\}$, onde $p_{\tilde{x}_{t}}$ é o número de partições do universo de discurso associado à variável linguística $\tilde{x}_{t}$. As matrizes $A_{i}$ e $B_{i}$ representam os parâmetros do $i$-ésimo modelo local do sistema fuzzy. A variável $\mathbf{x}(t)$ representa o vetor de estado do subsistema, enquanto que $\mathbf{u}(t)$ representa o vetor de entrada. $\mathrm{O}$ consequente, deste modo, consiste de uma equação de estado que representa um submodelo linear em um ponto de operação do sistema. O grau de ativação $h_{i}$ para a regra $R^{i}$ é dado pelo operador de conjunção norma-t:

$$
h_{i}=\gamma_{F_{j \mid \tilde{x}_{1}^{*}}}^{i} \wedge \gamma_{F_{j \mid \tilde{x}_{2}^{*}}^{*}}^{i} \wedge \ldots \wedge \gamma_{F_{j \mid \tilde{x}_{n}^{*}}}^{i}
$$

onde $\tilde{x}_{t}^{*}$ é algum ponto em $U_{\tilde{x}_{t}^{*}}$. O grau de ativação normalizado para a regra $i$ é definido como:

$$
\lambda_{i}(\tilde{\mathbf{x}})=\frac{h_{i}(\tilde{\mathbf{x}})}{\sum_{r=1}^{l} h_{r}(\tilde{\mathbf{x}})}
$$

Esta normalização implica que

$$
\sum_{i=1}^{l} \lambda_{i}(\tilde{\mathbf{x}})=1
$$

A resposta do modelo T-S é uma soma ponderada dos consequentes, isto é, uma combinação convexa dos modelos locais:

$$
\dot{\tilde{\mathbf{x}}}(t)=\sum_{i=1}^{l} \lambda_{i}(\tilde{\mathbf{x}})\left(A_{i} \mathbf{x}(t)+B_{i} \mathbf{u}(t)\right)
$$

Tal modelo pode ser visto como um sistema linear variante nos parâmetros $(L P V)$. Neste sentido, um modelo T-S pode ser considerado como uma mapeamento do espaço do antecedente (entrada) à região convexa (politopo) no espaço dos submodelos locais definidos pelos parâmetros do consequente. Esta propriedade simplifica a análise de modelos TS num contexto de sistema linear robusto tanto para identificação como para projeto de controladores com características de malhafechada desejadas ou análise de estabilidade.

\subsection{Análise do atraso puro de tempo}

O atraso puro de tempo é um fenômeno presente na maioria dos sistemas dinâmicos reais e sua análise é importante em virtude de seu efeito redutor de estabilidade em malhas de controle. Ele designa o tempo que um sistema leva para responder a uma dada entrada. Um atraso de tempo de $\tau_{d}$ unidades de tempo pode ser representando no domínio da frequência pelo termo $e^{-\tau_{d} s}$, o qual pode ser representado de forma aproximada como a razão entre dois polinômios em $s$ (aproximações de Padé):

$$
e^{-\tau_{d} s} \approx R_{n}(s)=\frac{Q_{n}\left(-\tau_{d} s\right)}{Q_{n}\left(\tau_{d} s\right)}
$$

sendo

$$
Q_{n}\left(\tau_{d} s\right)=\sum_{j=0}^{n} \frac{(n+j) !}{j !(n-j) !}\left(\tau_{d} s\right)^{n-j}
$$

Neste trabalho, em particular, consideraremos a aproximação de segunda ordem $(n=2)$, a qual fornece o termo

$$
R_{2}(s)=\frac{\tau_{d}^{2} s^{2}-6 \tau_{d} s+12}{\tau_{d}^{2} s^{2}+6 \tau_{d} s+12}
$$

o qual será multiplicado pela função de transferência que representa o $i$-ésimo submodelo local do modelo fuzzy para então ser feita a conversão para espaço de estados, resultando na estrutura de submodelo apresentada na seção 2.3.

\subsection{Problema de controle linear quadrático fuzzy no espaço de estados}

Neste artigo, o problema de controle linear quadrático fuzzy no espaço de estados apresenta a seguinte configuração:

$$
\begin{aligned}
\dot{\mathbf{x}}(t) & =\mathbf{A}_{i} \mathbf{x}(t)+\mathbf{B}_{i} u(t) \\
y(t) & =\mathbf{C}_{i} \mathbf{x}(t) \\
u(t) & =-\mathbf{K}_{i} \mathbf{x}(t)+k_{I}^{i} \psi(t) \\
\dot{\psi}(t) & =r(t)-y(t)=r(t)-\mathbf{C}_{i} \mathbf{x}(t)
\end{aligned}
$$

onde 
$\psi(t) \quad$ - saída do integrador

$r(t)$ - sinal de referência

Dada a entrada de referência $r(t)$ aplicada em $t=0$, para $t>0$, as equações (9) a (12) podem ser combinadas e descritas sob a forma de uma única equação matricial:

$\left[\begin{array}{c}\dot{\mathbf{x}}(t) \\ \dot{\psi}(t)\end{array}\right]=\left[\begin{array}{rr}\mathbf{A}_{i} & \mathbf{0} \\ -\mathbf{C}_{i} & 0\end{array}\right]\left[\begin{array}{c}\mathbf{x}(t) \\ \psi(t)\end{array}\right]+\left[\begin{array}{c}\mathbf{B}_{i} \\ 0\end{array}\right] u(t)+\left[\begin{array}{l}\mathbf{0} \\ 1\end{array}\right] r(t)$

Para determinado instante $t_{f}$ considerado pode-se escrever:

$\left[\begin{array}{c}\dot{\mathbf{x}}\left(t_{f}\right) \\ \dot{\psi}\left(t_{f}\right)\end{array}\right]=\left[\begin{array}{rr}\mathbf{A}_{i} & 0 \\ -\mathbf{C}_{i} & 0\end{array}\right]\left[\begin{array}{l}\mathbf{x}\left(t_{f}\right) \\ \psi\left(t_{f}\right)\end{array}\right]+\left[\begin{array}{c}\mathbf{B}_{i} \\ 0\end{array}\right] u\left(t_{f}\right)+\left[\begin{array}{l}\mathbf{0} \\ 1\end{array}\right] r\left(t_{f}\right)$

Considerando que a entrada $r(t)$ é suficientemente lenta para $t>0$, subtraindo-se a equação (14) da (13), obtem-se:

$\left[\begin{array}{c}\dot{\mathbf{x}}\left(t_{f}\right)-\dot{\mathbf{x}}(t) \\ \dot{\psi}\left(t_{f}\right)-\dot{\psi}(t)\end{array}\right]=\left[\begin{array}{rr}\mathbf{A}_{i} & 0 \\ -\mathbf{C}_{i} & 0\end{array}\right]\left[\begin{array}{c}\mathbf{x}\left(t_{f}\right)-\mathbf{x}(t) \\ \psi\left(t_{f}\right)-\psi(t)\end{array}\right]+\left[\begin{array}{c}\mathbf{B}_{i} \\ 0\end{array}\right]\left(u\left(t_{f}\right)-u(t)\right)$

Seja

$$
\begin{aligned}
\mathbf{x}(t)-\mathbf{x}\left(t_{f}\right) & =\mathbf{x}_{e}(t) \\
\psi(t)-\psi\left(t_{f}\right) & =\psi_{e}(t) \\
u(t)-u\left(t_{f}\right) & =u_{e}(t)
\end{aligned}
$$

Assim, a equação (15) pode ser escrita da forma:

$$
\left[\begin{array}{c}
\dot{\mathbf{x}}_{e}(t) \\
\dot{\psi}_{e}(t)
\end{array}\right]=\left[\begin{array}{rr}
\mathbf{A}_{i} & \mathbf{0} \\
-\mathbf{C}_{i} & 0
\end{array}\right]\left[\begin{array}{l}
\mathbf{x}_{e}(t) \\
\psi_{e}(t)
\end{array}\right]+\left[\begin{array}{c}
\mathbf{B}_{i} \\
0
\end{array}\right] u_{e}(t)
$$

onde

$$
u_{e}(t)=-\mathbf{K}_{i} \mathbf{x}_{e}(t)+k_{I}^{i} \psi_{e}(t)
$$

Definindo-se o vetor de erro e $(t)$ :

$$
\mathbf{e}(t)=\left[\begin{array}{l}
\mathbf{x}_{e}(t) \\
\psi_{e}(t)
\end{array}\right]
$$

as equações (19) e (20) resultam, respectivamente, em:

$$
\begin{aligned}
\dot{\mathbf{e}}(t) & =\hat{\mathbf{A}}_{i} \mathbf{e}(t)+\hat{\mathbf{B}}_{i} u_{e}(t) \\
u_{e}(t) & =-\hat{\mathbf{K}}_{i} \mathbf{e}(t)
\end{aligned}
$$

onde

$$
\hat{\mathbf{A}}_{i}=\left[\begin{array}{rr}
\mathbf{A}_{i} & 0 \\
-\mathbf{C}_{i} & 0
\end{array}\right], \quad \hat{\mathbf{B}}_{i}=\left[\begin{array}{c}
\mathbf{B}_{i} \\
0
\end{array}\right], \quad \hat{\mathbf{K}}_{i}=\left[\mathbf{K}_{i} \mid-k_{I}^{i}\right]
$$

Subtituindo-se a equação (23) na (22), a equação de estado do erro pode ser obtida:

$$
\dot{\mathbf{e}}(t)=\left(\hat{\mathbf{A}}_{i}-\hat{\mathbf{B}}_{i} \hat{\mathbf{K}}_{j}\right) \mathbf{e}(t)
$$

Analisando-se a equação (25), observa-se que uma vez especificados os autovalores desejados da matriz $\hat{\mathbf{A}}_{i}-\hat{\mathbf{B}}_{i} \hat{\mathbf{K}}_{j}$, a matriz $\hat{\mathbf{K}}_{i}$ e consequentemente $\mathbf{K}_{i}$ e $k_{I}^{i}$ podem ser determinados pela técnica de alocação de pólos. A técnica LQR, por outro lado, também permite a determinação da matriz $\hat{\mathbf{K}}_{i}$. A combinação das duas técnicas em um contexto fuzzy, como será mostrado, permite a alocação ótima dos pólos, caracterizando o aspecto multiobjetivo da abordagem proposta. Seja, então, o problema de controle quadrático ótimo, que possibilita minimizar o índice de desempenho dado por

$$
J=\int_{0}^{\infty}\left(\mathbf{e}^{T} \mathbf{Q} \mathbf{e}+\mathbf{u}_{\mathbf{e}}^{T} \mathbf{R} \mathbf{u}_{e}\right) d t
$$

inerente ao sistema descrito pelas equações (22) e (23). Na equação (26), $\mathbf{Q}$ e $\mathbf{R}$ são matrizes de ponderação sobre o estado do erro $\mathbf{e}(t)$ e a entrada $\mathbf{u}_{e}(t)$, respectivamente. A matriz $\mathbf{Q}$ é uma matriz hermitiana definida ou semidefinida positiva ou ainda real simétrica, e a matriz $\mathbf{R}$ é hermitiana definida positiva ou real simétrica. Considerando que a matriz $\hat{\mathbf{A}}_{i}-\hat{\mathbf{B}}_{i} \hat{\mathbf{K}}_{j}$ é estável (autovalores com parte real negativa), é possível obter a matriz de ganho de controle por realimentação de estado $\hat{\mathbf{K}}_{i}$, uma vez que se solucione, para a matriz $\mathbf{P}$, a equação algébrica de Riccati:

$$
\hat{\mathbf{A}}_{i}^{T} \mathbf{P}+\mathbf{P} \hat{\mathbf{A}}_{i}-\mathbf{P} \hat{\mathbf{B}}_{i} \mathbf{R}^{-1} \hat{\mathbf{B}}_{i}^{T} \mathbf{P}+\mathbf{Q}=\mathbf{0}
$$

Uma vez obtida a matriz $\mathbf{P}$, da solução da equação (27), a matriz de ganho $\hat{\mathbf{K}}_{i}$ é calculada por:

$$
\hat{\mathbf{K}}_{i}=\mathbf{R}^{-1} \hat{\mathbf{B}}_{i}^{T} \mathbf{P}
$$

Considere o seguinte estrutura de submodelo no espaço de estados na forma canônica de controle:

$$
\begin{aligned}
\dot{\mathbf{x}}(t) & =\mathbf{A}_{i} \mathbf{x}(t)+\mathbf{B}_{i} u(t) \\
y(t) & =\mathbf{C}_{i} \mathbf{x}(t)
\end{aligned}
$$

Considerando que será utilizado um modelo de segunda ordem para representar o submodelo local e também segunda ordem para a aproximação de Padé, estabelecemos as seguintes matrizes da equação (29): 


$$
\begin{gathered}
\mathbf{B}_{i}=\left[\begin{array}{c}
0 \\
0 \\
0 \\
1
\end{array}\right], \\
\mathbf{C}_{i}=\left[\begin{array}{llll}
\frac{12 b_{1}^{i}}{a_{1}^{i} \tau_{d}{ }^{2}} & -\frac{6 b_{1}^{i}}{a_{1}^{i} \tau_{d}} & \frac{b_{1}^{i}}{a_{1}^{i}} & 0
\end{array}\right]
\end{gathered}
$$

As matrizes $\mathbf{Q}$ e $\mathbf{R}$ são definidas como

$$
\begin{gathered}
\mathbf{Q}=\left[\begin{array}{lllll}
\mu & 0 & 0 & 0 & 0 \\
0 & 1 & 0 & 0 & 0 \\
0 & 0 & 1 & 0 & 0 \\
0 & 0 & 0 & 1 & 0 \\
0 & 0 & 0 & 0 & 1
\end{array}\right] \\
\mathbf{R}=[\beta]
\end{gathered}
$$

onde $\mu \geq 0$ e $\beta>0$. Os parâmetros $\mu$ e $\beta$ estão diretamente relacionados à alocação ótima dos pólos uma vez que seja possível sua determinação.

Com base na equação (24) e nas equações (30), (31) e (32) as matrizes $\hat{\mathbf{A}}_{i}$ e $\hat{\mathbf{B}}_{i}$ são obtidas. A matriz $\mathbf{P}$ a ser obtida, consiste numa matriz real simétrica:

$$
\mathbf{P}=\left[\begin{array}{lllll}
p_{11} & p_{12} & p_{13} & p_{14} & p_{15} \\
p_{12} & p_{22} & p_{23} & p_{24} & p_{25} \\
p_{13} & p_{23} & p_{33} & p_{34} & p_{35} \\
p_{14} & p_{24} & p_{34} & p_{44} & p_{45} \\
p_{15} & p_{25} & p_{35} & p_{45} & p_{55}
\end{array}\right]
$$

Substituindo-se as matrizes $\hat{\mathbf{A}}_{i}$ e $\hat{\mathbf{B}}_{i}$ obtidas e as equações (33) a (35) na equação (27), obtém-se o seguinte sistema de equações:

$$
\begin{aligned}
& \mu-\frac{p_{14}{ }^{2}}{\beta}-\frac{24 p_{14}}{a_{1}^{i} \tau_{d}^{2}}-\frac{24 b_{1}^{i} p_{15}}{a_{1}^{i} \tau^{2}}=0 \\
& p_{11}-\frac{p_{14} p_{24}}{\beta}-\frac{12 p_{24}}{a_{1}^{i} \tau_{d}^{2}}+\frac{6 b_{1}^{i} p_{15}}{a_{1}^{i} \tau_{d}}-\frac{12 b_{1}^{i} p_{25}}{a_{1}^{i} \tau_{d}^{2}}-\frac{6 p_{14}\left(2 a_{2}^{i}+\tau_{d}\right)}{a_{1}^{i} \tau^{2}}=0 \\
& p_{12}-p_{14}\left(\frac{12}{\tau_{d}{ }^{2}}+\frac{6 a_{2}^{i}+\tau_{d}}{a_{1}^{i} \tau_{d}}\right)-\frac{p_{14} p_{34}}{\beta}-\frac{12 p_{34}}{a_{1}^{i} \tau_{d}{ }^{2}}-\frac{b_{1}^{i} p_{15}}{a_{1}^{i}}-\frac{12 b_{1}^{i} p_{35}}{a_{1}^{i} \tau_{d}{ }^{2}}=0 \\
& p_{13}-p_{14}\left(\frac{a_{2}^{i}}{a_{1}^{i}}+\frac{6}{\tau_{d}}\right)-\frac{p_{14} p_{44}}{\beta}-\frac{12 p_{44}}{a_{1}^{i} \tau_{d}{ }^{2}}-\frac{12 b_{1}^{i} p_{45}}{a_{1}^{i} \tau_{d}{ }^{2}}=0 \\
& -\frac{p_{14} p_{45}}{\beta}-\frac{12 p_{45}}{a_{1}^{i} \tau_{d}^{2}}-\frac{12 b_{1}^{i} p_{55}}{a_{1}^{i} \tau_{d}^{2}}=0 \\
& 2 p_{12}-\frac{p_{24}{ }^{2}}{\beta}+\frac{12 b_{1}^{i} p_{25}}{a_{1}^{i} \tau_{d}}-\frac{12 p_{24}\left(2 a_{2}^{i}+\tau d\right)}{a_{1}^{i} \tau_{d}^{2}}+1=0 \\
& p_{13}+p_{22}-p_{24}\left(\frac{12}{\tau_{d}^{2}}+\frac{6 a_{2}^{i}+\tau_{d}}{a_{1}^{i} \tau_{d}}\right)-\frac{p_{24} p_{34}}{\beta}-\frac{b_{1}^{i} p_{25}}{a_{1}^{i}}+\frac{6 b_{1}^{i} p_{35}}{a_{1}^{i} \tau_{d}} \\
& -\frac{6 p_{34}\left(2 a_{2}^{i}+\tau_{d}\right)}{a_{1}^{i} \tau_{d}^{2}}=0
\end{aligned}
$$

$$
\begin{gathered}
p_{14}+p_{23}-p_{24}\left(\frac{a_{2}^{i}}{a_{1}^{i}}+\frac{6}{\tau_{d}}\right)-\frac{p_{24} p_{44}}{\beta}+\frac{6 b_{1}^{i} p_{45}}{a_{1}^{i} \tau_{d}}-\frac{6 p_{44}\left(2 a_{2}^{i}+\tau_{d}\right)}{a_{1}^{i} \tau_{d}^{2}}=0 \\
p_{15}-\frac{p_{24} p_{45}}{\beta}+\frac{6 b_{1}^{i} p_{55}}{a_{1}^{i} \tau_{d}}-\frac{6 p_{45}\left(2 a_{2}^{i}+\tau_{d}\right)}{a_{1}^{i} \tau_{d}{ }^{2}}=0
\end{gathered}
$$

$$
\begin{gathered}
2 p_{23}-2 p_{34}\left(\frac{12}{\tau_{d}{ }^{2}}+\frac{6 a_{2}^{i}+\tau_{d}}{a_{1}^{i} \tau_{d}}\right)-\frac{p_{34}{ }^{2}}{\beta}-\frac{2 b_{1}^{i} p_{35}}{a_{1}^{i}}+1=0 \\
p_{24}+p_{33}-p_{34} \\
\left(\frac{a_{2}^{i}}{a_{1}^{i}}+\frac{6}{\tau_{d}}\right)-p_{44}\left(\frac{12}{\tau_{d}^{2}}+\frac{6 a_{2}^{i}+\tau_{d}}{a_{1}^{i} \tau_{d}}\right)+ \\
-\frac{p_{34} p_{44}}{\beta}-\frac{b_{1}^{i} p_{45}}{a_{1}^{i}}=0
\end{gathered}
$$

$$
\begin{gathered}
p_{25}-p_{45}\left(\frac{12}{\tau_{d}{ }^{2}}+\frac{6 a_{2}^{i}+\tau_{d}}{a_{1}^{i} \tau_{d}}\right)-\frac{p_{34} p_{45}}{\beta}-\frac{b_{1}^{i} p_{55}}{a_{1}^{i}}=0 \\
2 p_{34}-2 p_{44}\left(\frac{a_{2}^{i}}{a_{1}^{i}}+\frac{6}{\tau_{d}}\right)-\frac{p_{44}{ }^{2}}{\beta}+1=0 \\
p_{35}-p_{45}\left(\frac{a_{2}^{i}}{a_{1}^{i}}+\frac{6}{\tau_{d}}\right)-\frac{p_{44} p_{45}}{\beta}=0 \\
1-\frac{p_{45}{ }^{2}}{\beta}=0
\end{gathered}
$$

Após a solução numérica do sistema descrito pelas equações de (36) a (50), para a matriz $\mathbf{P}$, matriz $\hat{\mathbf{K}}_{i}$ é calculada a partir de (28)

$$
\hat{\mathbf{K}}_{i}=\mathbf{R}^{-1} \hat{\mathbf{B}}_{i}^{T} \mathbf{P}=\left[\begin{array}{lllll}
\frac{p_{14}}{\beta} & \frac{p_{24}}{\beta} & \frac{p_{34}}{\beta} & \frac{p_{44}}{\beta} & \frac{p_{45}}{\beta}
\end{array}\right]
$$

A equação característica do sistema de controle, por sua vez, é dada por:

$$
\begin{gathered}
\left|s \mathbf{I}_{5}-\hat{\mathbf{A}}_{i}+\hat{\mathbf{B}}_{i} \hat{\mathbf{K}}_{i}\right|= \\
s^{5}+\left(\frac{a_{1}^{i} p_{44} \tau_{d}^{2}+6 a_{1}^{i} \beta \tau_{d}+a_{2}^{i} \beta \tau_{d}^{2}}{a_{1}^{i} \beta \tau_{d}{ }^{2}}\right) s^{4}+ \\
\left(\frac{12 a_{1}^{i} \beta+\beta \tau_{d}^{2}+a_{1}^{i} p_{34} \tau_{d}^{2}+6 a_{2}^{i} \beta \tau_{d}}{a_{1}^{i} \beta \tau_{d}{ }^{2}}\right) s^{3}+ \\
\left(\frac{12 a_{2}^{i} \beta+6 \beta \tau_{d}+a_{1}^{i} p_{24} \tau_{d}{ }^{2}-b_{1}^{i} p_{45} \tau_{d}{ }^{2}}{a_{1}^{i} \beta \tau_{d}{ }^{2}}\right) s^{2}+ \\
\left(\frac{a_{1}^{i} p_{14} \tau_{d}{ }^{2}+6 b_{1}^{i} p_{45} \tau_{d}+12 \beta}{a_{1}^{i} \beta \tau_{d}{ }^{2}}\right) s-\frac{12 b_{1}^{i} p_{45}}{a_{1}^{i} \beta \tau_{d}{ }^{2}}=0
\end{gathered}
$$

Após a identificação do sistema a ser controlado são obtidos todos os parâmetros dos submodelos locais. Daí procede-se com a varredura de valores factíveis de $\mu$ e $\beta$ para solução da equação algébrica de Riccati, para a matriz $\mathbf{P}$ e a consequente determinação das matrizes de ganho de controle por realimentação de estado para cada um dos submodelos e a base de dados de pólos factíveis. 


\section{Resultados de Discussão}

\subsection{Plataforma de controle virtual/eletrônica}

Neste trabalho, o processo de aquisição de dados para identificação bem como a implementação do método de controle proposto são realizados em uma plataforma de controle virtual/eletrônica desenvolvida com base no software LabVIEW (Laboratory Virtual Instrument Engineering Workbench) e na controladora CompactRIO 9073, ambos da National Instruments. Os principais módulos integrados na controladora e utilizados na plataforma são: NI 9219 (entrada analógica) e NI 9263 (saída analógica). Dado que a aplicação será realizada em um processo térmico, a plataforma conta ainda com o sensor de temperatura LM 35, o qual fornece uma tensão proporcional à temperatura a qual está submetido $\left(10 \mathrm{mV} /{ }^{\circ} \mathrm{C}\right)$ e um circuito atuador baseado no circuito integrado TCA 785, capaz de variar o ângulo de disparo de tiristores pela aplicação de uma tensão contínua.

\subsection{Modelagem fuzzy T-S do processo térmico}

Esta etapa de modelagem fuzzy T-S está baseada em dados experimentais de entrada e saída (identificação) do processo térmico. Na Figura 1 é mostrado o sinal de tensão eficaz $\left(V_{r m s}\right)$ aplicado à entrada do sistema e a respectiva saída (Temperatura - ${ }^{\circ} \mathrm{C}$ ) obtida. Este conjunto de dados, constituído de 29942 pontos a um tempo de amostragem de 17 milissegundos foi utilizado para identificação.
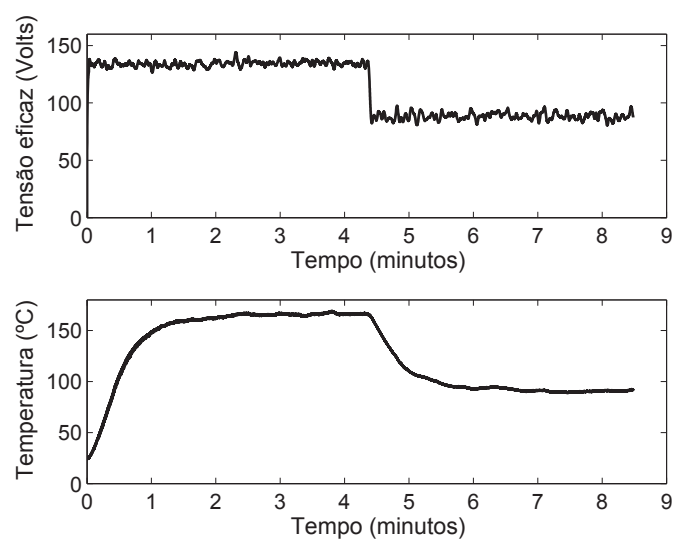

Figura 1: Teste dinâmico para identificação aplicado ao processo térmico. A tensão alternada, elucidada pelo seu valor eficaz, é aplicada ao processo térmico cuja resposta temporal é temperatura, em graus Celsius.

A condição inicial de temperatura para este conjunto de dados é de $24.2951{ }^{\circ} \mathrm{C}$. O atraso de tempo do processo térmico foi obtido aplicado-se correlação cruzada nos dados de entrada e saída, como mostra a Figura 2. O valor do atraso de tempo é determinado a partir do atraso correspondente ao ponto de máximo do gráfico (966), considerando o tempo de amostragem de 17 milissegundos, fornecendo $\tau_{d}=1.6422 \mathrm{~s}$.

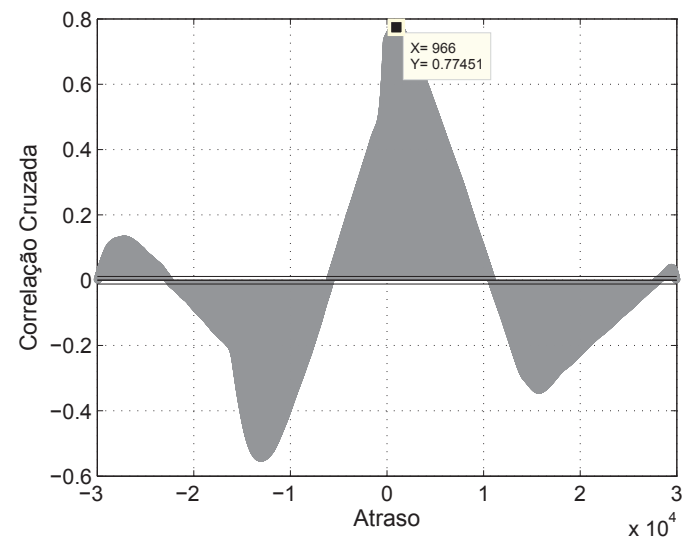

Figura 2: Função de correlação cruzada aplicada aos sinais de entrada e saída do processo térmico.

O algoritmo de agrupamento fuzzy c-means com método dos mínimos quadrados para estimação dos parâmetros do consequente foi aplicado ao sinal de saída e o seguinte modelo fuzzy T-S foi obtido:

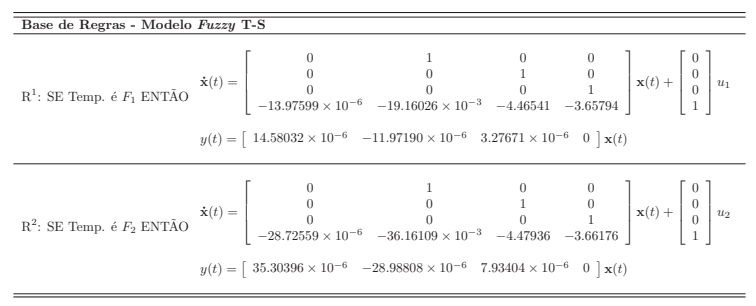

Tabela 1: Base de regras do modelo fuzzy TakagiSugeno para processo térmico. 'Temp.' indica temperatura.

onde

$$
\begin{aligned}
& F_{1}\left(\text { Temp., a,b) }\left.\right|_{(a=120 ; b=132.5)}=\right. \\
& \left\{\begin{array}{cc}
1, & \text { Temp. } \leq a \\
1-2\left(\frac{\text { Temp. }-a}{b-a}\right)^{2}, & a \leq \text { Temp. } \leq \frac{a+b}{2} \\
2\left(\frac{\text { Temp. }-a}{b-a}\right)^{2}, & \frac{a+b}{2} \leq \text { Temp. } \leq b \\
0, & \text { Temp. } \geq b
\end{array}\right.
\end{aligned}
$$

e $F_{2}=1-F_{1}$.

A fato de haver presença de ruído nos sinais de entrada e saída e considerando a formulação no tempo contínuo na qual aparecem termos derivativos do sinal de saída na matriz de regressores (que aplificam o ruído), o algoritmo dos mínimos quadrados tem um desempenho insatisfatório na estimação dos parâmetros do consequente. Deste modo, o modelo fuzzy T-S identificado foi submetido a um processo de otimização por algoritmo genético (AG). Os parâmetros do AG foram os 
seguintes: 1. tamanho do cromossomo - 4 (ganhos dos dois submodelos lineares e intensificadores das funções de pertinência); 2. tamanho da população - 50; 3. número de gerações - 300; 4. probabilidade de crossover aritmético - 85\%; 5 . probabilidade de seleção natural - 50\%; 6 . probabilidade de mutação - 50\%. A função de custo do AG (fitness) é baseada na norma da diferença entre os vetores de dados da saída real $\left(y_{\text {real }}(m)\right)$ e da saída do modelo $\left(y_{\text {modelo }}(m)\right)$. Na Figura 3 é mostrada a otimização do modelo T-S por meio do AG. A norma do modelo fuzzy T-S identificado é de 3653.706. A norma do modelo fuzzy T-S otimizado, por sua vez, é de 1108.212.

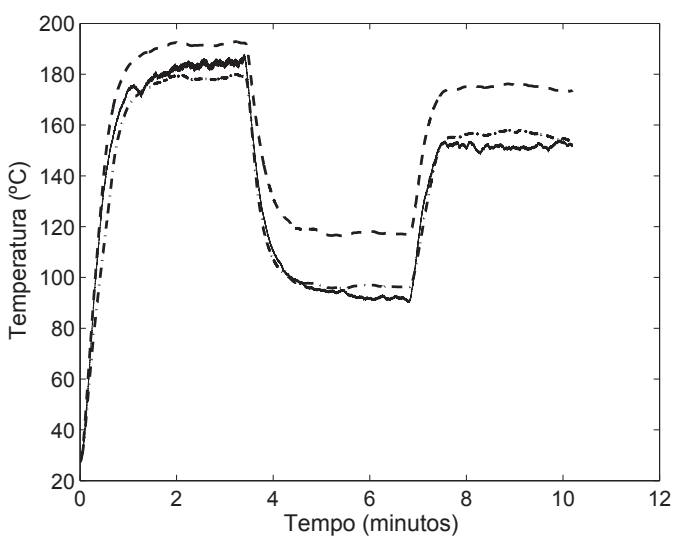

Figura 3: Otimização via algoritmo genético do modelo fuzzy T-S. A linha sólida indica a saída do processo térmico, a linha tracejada indica a saída do modelo T-S identificado e a linha traço e ponto indica a saída do modelo T-S otimizado.

Com a otimização, o ganhos dos submodelos lineares 1 e 2 passaram a ser, respectivamente, $919.874482693833 \times 10^{-3}$ e 1.14203009259494 . Os intensificadores $m_{1}$ e $m_{2}$ das funções de pertinência, que realizam as operação $\left(F_{1}\right)^{m_{1}}$ e $\left(F_{2}\right)^{m_{2}}$, ficaram com os valores $m_{1}=991.157707944464 \times$ $10^{-3}$ e $m_{2}=1.00127740549514$. Um intensificador $m_{i}$, se $m_{i}>1$, a operação resultante é de contração ${ }^{1}$ sobre a função de pertinência. Caso $0<m_{i}<1$, a operação é de dilatação.

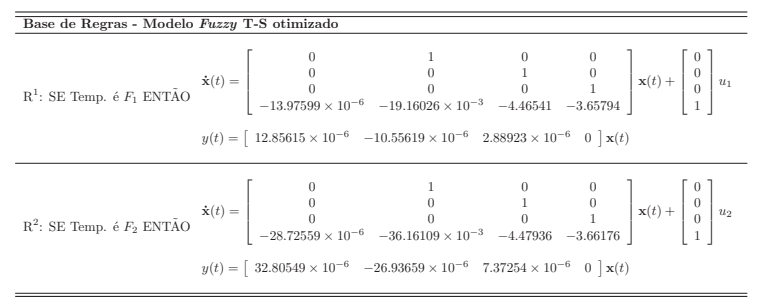

Tabela 2: Base de regras do modelo fuzzy Takagi-Sugeno (otimizado) para processo térmico. 'Temp.' indica temperatura.

\footnotetext{
${ }^{1}$ Aqui, a operação de contração, como também de expansão é referente à variância da função.
}

Na Figura 4 são mostradas diferentes respostas temporais do sistema de controle fuzzy T-S baseado na metodologia proposta para diferentes alocações de pólos.

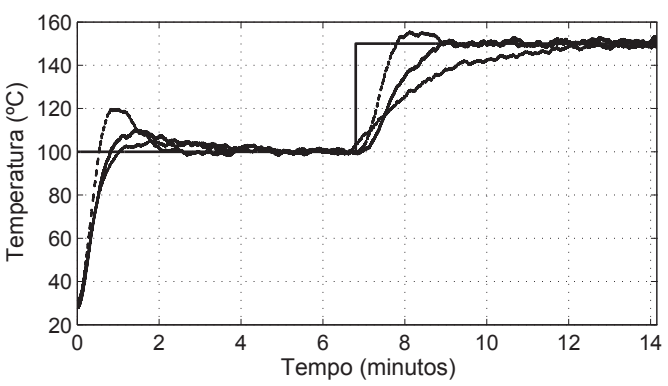

Figura 4: Repostas temporais do sistema de controle fuzzy. Os pólos correspondentes à resposta transitória na região intermediária das três curvas são: $-96.715,-845.47 \times 10^{-3} \pm 554.3361 \times 10^{-3} j$, $-319.964 \times 10^{-3}$ e $-2.03 \times 10^{-6}$.

\section{Conclusões}

A metodologia proposta garantiu um controlador ótimo com desempenho temporal satisfatório baseado em pólos adequadamente alocados e cujo projeto leva em conta o atraso puro de tempo da planta a ser controlada. Resultados experimentais para controle LQR fuzzy de temperatura, via plataforma de aquisição de dados, em tempo real, mostraram a eficiência e a flexibilidade de projeto.

\section{Agradecimentos}

Os autores agradecem ao Conselho Nacional de Desenvolvimento Científico e Tecnológico (CNPq) pelo fomento à pesquisa.

\section{Referências}

Babuška, R. (1998). Fuzzy Modeling for Control, Kluwer Academic Publishers, Massachusetts. DOI: 10.1007/978-94-011-4868-9

Jahromi, A. and Zabihollah, A. (2010). Linear quadratic regulator and fuzzy controller application in full-car model of suspension system with magnetorheological shock absorber, pp. 522-528.

Lendek, Z., Babuška, R. and De Schutter, B. (2009). Stability of cascaded fuzzy systems and observers, Fuzzy Systems, IEEE Transactions on 17(3): 641-653.

Michels, K., Klawonn, F., Kruse, R. and Nurnberger, A. (2006). Fuzzy Control: Fundamentals, Stability and Design of Fuzzy Controllers, Springer. 